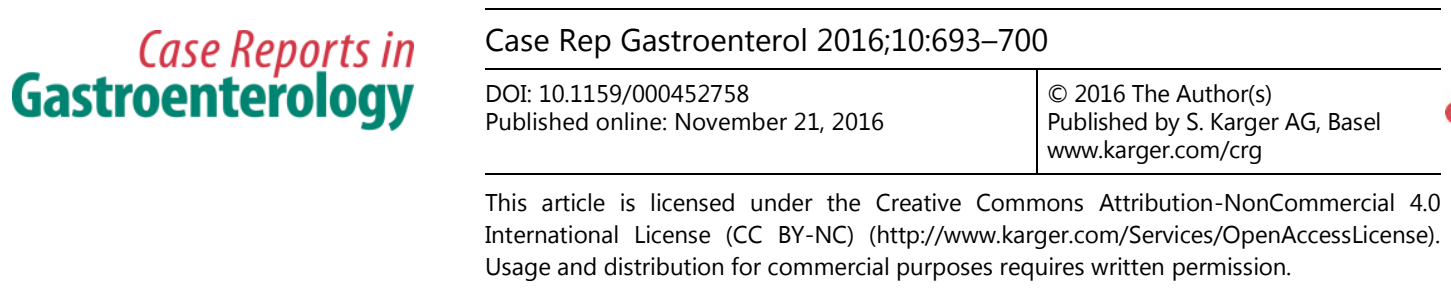

\title{
Two Cases of Rectal Neuroendocrine Tumor Resection Combined with Dissection of the Circular Muscle Layer Using the Endoscopic Submucosal Dissection Technique
}

\author{
Kumpei Honjo ${ }^{a}$ Kazumasa Kure ${ }^{a}$ Ryosuke Ichikawa ${ }^{a} H_{i s a s h i ~ R o}{ }^{a}$ \\ Rina Takahashi $^{\mathrm{a}}$ Koichiro Niwa $^{\mathrm{a}}$ Shun Ishiyama $^{\mathrm{a}}$ Kiichi Sugimoto $^{\mathrm{a}}$ \\ Hirohiko Kamiyama $^{\text {a }}$ Makoto Takahashi $^{\mathrm{a}}$ Yutaka Kojima ${ }^{\mathrm{a}}$ \\ Michitoshi Goto $^{a}$ Yuichi Tomiki ${ }^{a}$ Kazuhiro Sakamoto ${ }^{a}$ Yuki Fukumura ${ }^{b}$ \\ Takashi Yao ${ }^{\text {b }}$ \\ ${ }^{a}$ Department of Coloproctological Surgery, Juntendo University Faculty of Medicine, \\ Tokyo, Japan; ${ }^{b}$ Department of Human Pathology, Juntendo University Faculty of Medicine, \\ Tokyo, Japan
}

\section{Keywords}

Neuroendocrine tumor - Endoscopic submucosal dissection - Endoscopic mucosal resection with a ligation device

\begin{abstract}
Generally, lesions of rectal neuroendocrine tumors (NETs) $10 \mathrm{~mm}$ or smaller are less malignant and are indicated for endoscopic therapy. However, the vertical margin may remain positive after conventional endoscopic mucosal resection (EMR) because NETs develop in a way similar to submucosal tumors (SMTs). The usefulness of EMR with a ligation device, which is modified EMR, and endoscopic submucosal dissection (ESD) was reported, but no standard treatment has been established. We encountered 2 patients in whom rectal NETs were completely resected by combined dissection and resection of the circular muscle layer using the ESD technique. Case 1 was an 8-mm NET of the lower rectum. Case 2 was NET of
\end{abstract}


the lower rectum treated with additional resection for a positive vertical margin after EMR. In both cases, the circular muscle layer was dissected applying the conventional ESD technique, followed by en bloc resection while conserving the longitudinal muscle layer. No problems occurred in the postoperative course in either case. Rectal NETs are observed in the lower rectum in many cases, and it is less likely that intestinal perforation by endoscopic therapy causes peritonitis. The method employed in these cases, namely combined dissection and resection of the circular muscle layer using the ESD technique, can be performed relatively safely, and it is possible to ensure negativity of the vertical margin. In addition, it may also be useful for additional treatment of cases with a positive vertical margin after EMR.

(C) 2016 The Author(s)

Published by S. Karger AG, Basel

\section{Introduction}

Neuroendocrine neoplasms are composed of cells with a neuroendocrine phenotype. The 2010 WHO classification distinguishes between well-differentiated and poorly differentiated neoplasms. All well-differentiated neoplasms are called neuroendocrine tumors (NETs), and all poorly differentiated neoplasms are termed neuroendocrine carcinomas.

Approximately $10-15 \%$ of all NETs occur in the rectum [1]. Rectal NETs $<10 \mathrm{~mm}$ in size have a very low risk of lymph node metastasis. Thus, 10 -mm or smaller lesions were endoscopically resected in many case reports. However, the vertical margin remains positive after conventional endoscopic therapy because NETs develop in a submucosal tumor (SMT)like way, for which circumferential submucosal incision prior to endoscopic mucosal resection (EMR), EMR using a cap-fitted endoscope, and EMR with a ligation device (EMR-L) have been devised [2-4]. The usefulness of endoscopic submucosal dissection (ESD) has also recently been reported [5-7], but no standard treatment has been established.

We completely resected rectal NETs by the combined dissection and resection of the circular muscle layer using the ESD technique in 2 patients to ensure negativity of the vertical margin.

\section{Case Presentations}

Case 1 was a female patient in her 50s. She consulted a physician for positive fecal occult blood, and an 8-mm SMT slightly more yellowish than the surrounding mucosa was present in the lower rectum (Fig. 1a). NET classified as G1 was diagnosed on biopsy, and the patient was referred to our hospital. Resection by ESD using an electric knife (Dual Knife; Olympus, Tokyo, Japan) was tried. To achieve negative vertical margin, dissection was advanced to the circular muscle layer right below the tumor, and en bloc resection was performed. No perforation occurred because the longitudinal muscle layer was conserved (Fig. 1b, Fig. 1c). On histopathological examination, the tumor was a $10 \times 8-\mathrm{mm}$ G1 NET, and no lymphatic or venous invasion was noted (Fig. 1d, Fig. 1 e). Since the tumor and muscle layer were located closely, the negative resected margin could be achieved by resection of the circular muscle layer (Fig. 1f). In the postoperative course, no elevation of inflammatory reaction was detected on blood testing, and rectal perforation did not occur. The patient was discharged on the 3rd hospital day.

Case 2 was a male patient in his 60s. EMR was performed for a rectal polyp by a physician. The lesion measured $8 \mathrm{~mm}$ and was diagnosed as G1 NET on histopathological exami- 
nation. No lymphatic or venous invasion was observed, but the lateral and vertical margins were positive. Thus, the patient was referred to our hospital for additional treatment. On endoscopy, a white scar following EMR was present in the lower rectum (Fig. 2a). Marking was applied around the scar using an electric knife (Dual Knife; Olympus) (Fig. 2b). Since the vertical margin was positive, dissection was advanced to the circular muscle layer right below the scar, followed by en bloc resection. The longitudinal muscle layer was conserved (Fig. 2c, Fig. 2d). On histopathological examination, residual tumor was present, and combined resection of the circular muscle layer was performed (Fig. 2e, Fig. 2f). In the postoperative course, only a mild inflammatory reaction was detected on blood testing. No rectal perforation occurred, and the patient was discharged on the 2nd hospital day.

\section{Discussion}

Findings suggesting NETs include (1) a tumor diameter larger than $10 \mathrm{~mm}$; (2) infiltration in the muscle layer or deeper (muscular layer invasion); (3) surface concavity (central depression), and (4) lymphatic and venous invasion [8]. The tumor diameter is most strongly correlated with the lymph node metastasis rate. Malignancy is low when the tumor diameter is $10 \mathrm{~mm}$ or smaller, for which endoscopic therapy is frequently selected [8].

Histologically, NETs are basal mucosa-derived tumors (arising from the deep mucosal layer) and grow into the proper mucosal and submucosal layers. Therefore, the vertical margin may remain positive after conventional EMR, for which the usefulness of modified EMR to achieve a negative vertical margin has been reported [2-4]. In EMR-L, the base of NET is ligated with a rubber band (band ligation), and the lesion is cut below the rubber band. The treatment time is short, and a high complete resection rate can be achieved $[3,4]$. The outcome of the recently introduced ESD is also favorable, and a high complete resection rate has been reported, but the vertical margin remains positive on histopathological examination in a considerable number of cases $[2,5,6]$.

Generally, when the vertical margin is positive after endoscopic resection, additional surgical resection is considered necessary, but it may be over therapy with colostomy risk for less malignant NETs with a tumor of $10 \mathrm{~mm}$ or smaller. In addition, the prognosis of NETs that are $10 \mathrm{~mm}$ or smaller is favorable even tough lymphatic and venous invasion is observed [5, 9]. Therefore, even if the vertical margin is positive, local excision is useful for additional treatment, and intestinal resection accompanied by lymph node dissection is not useful. For local excision, transanal endoscopic microsurgery and minimally invasive transanal surgery were reported as safe and low-invasive treatment methods [10]. However, these techniques require specific skills and devices because they are surgical treatments.

The ESD skill is necessary to resect tumors with combined dissection and resection of the circular muscle layer using the ESD technique, but no other specific device is necessary and it can be applied within the range of endoscopic therapy. Its application is limited to the lower rectum with a low risk of perforation-induced peritonitis, but it is a low-invasive treatment method capable of ensuring negative vertical margin.

EMR-L and ESD are useful to treat NETs with a tumor diameter of $10 \mathrm{~mm}$ or less, but it may be desirable to regard EMR-L as the first choice because of its simple technique. Accordingly, the method with combined dissection of the circular muscle layer using the ESD technique introduced by us may be an option for additional treatment of vertical margin-positive cases after treatment of rectal NETs with EMR. 


\section{Case Reports in \\ Gastroenterology}

Case Rep Gastroenterol 2016;10:693-700

DOI: $10.1159 / 000452758$

(C) 2016 The Author(s). Published by S. Karger AG, Basel www.karger.com/crg

Honjo et al: Two Cases of Rectal NET Resection Combined with Dissection of the Circular Muscle Layer Using the Endoscopic Submucosal Dissection Technique

\section{References}

1 Jernman J, Välimäki MJ, Louhimo J, Haglund C, Arola J: The novel WHO 2010 classification for gastrointestinal neuroendocrine tumours correlates well with the metastatic potential of rectal neuroendocrine tumours. Neuroendocrinology 2012;95:317-324.

-2 Cheung DY, Choi SK, Kim HK, Kim SS, Chae HS, Seo KJ, Cho YS: Circumferential submucosal incision prior to endoscopic mucosal resection provides comparable clinical outcomes to submucosal dissection for well-differentiated neuroendocrine tumors of the rectum. Surg Endosc 2015;29:1500-1505.

- 3 Ono A, Fujii T, Saito Y, Matsuda T, Lee DT, Gotoda T, Saito D: Endoscopic submucosal resection of rectal carcinoid tumors with a ligation device. Gastrointest Endosc 2003;57:583-587.

- 4 Niimi K, Goto O, Fujishiro M, Kodashima S, Ono S, Mochizuki S, Asada-Hirayama I, Konno-Shimizu M, Mikami-Matsuda R, Minatsuki C, Yamamichi N, Koike K: Endoscopic mucosal resection with a ligation device or endoscopic submucosal dissection for rectal carcinoid tumors: an analysis of 24 consecutive cases. Dig Endosc 2012;24:443-447.

5 Zhong DD, Shao LM, Cai JT: Endoscopic mucosal resection vs endoscopic submucosal dissection for rectal carcinoid tumours: a systematic review and meta-analysis. Colorectal Dis 2013;15:283-291.

-6 Lee DS, Jeon SW, Park SY, Jung MK, Cho CM, Tak WY, Kweon YO, Kim SK: The feasibility of endoscopic submucosal dissection for rectal carcinoid tumors: comparison with endoscopic mucosal resection. Endoscopy 2010;42:647-651.

-7 Nakamura K, Osada M, Goto A, Iwasa T, Takahashi S, Takizawa N, Akahoshi K, Ochiai T, Nakamura N, Akiho H, Itaba S, Harada N, Iju M, Tanaka M, Kubo H, Somada S, Ihara E, Oda Y, Ito T, Takayanagi R: Short-and long-term outcomes of endoscopic resection of rectal neuroendocrine tumours: analyses according to the WHO 2010 classification. Scand J Gastroenterol 2016;51:448-455.

8 Kasuga A, Chino A, Uragami N, Kishihara T, Igarashi M, Fujita R, Yamamoto N, Ueno M, Oya M, Muto T: Treatment strategy for rectal carcinoids: a clinicopathological analysis of 229 cases at a single cancer institution. J Gastroenterol Hepatol 2012;27:1801-1807.

-9 Sekiguchi M, Sekine S, Sakamoto T, Otake Y, Nakajima T, Matsuda T, Taniguchi H, Kushima R, Ohe Y, Saito Y: Excellent prognosis following endoscopic resection of patients with rectal neuroendocrine tumors despite the frequent presence of lymphovascular invasion. J Gastroenterol 2015;50:1184-1189.

10 Kinoshita T, Kanehira E, Omura K, Tomori T, Yamada H: Transanal endoscopic microsurgery in the treatment of rectal carcinoid tumor. Surg Endosc 2007;21:970-974. 
\begin{tabular}{ll|l} 
Case Reports in & Case Rep Gastroenterol 2016;10:693-700 \\
\cline { 2 - 3 } Gast 10.1159/000452758 & $\begin{array}{l}\text { O 2016 The Author(s). Published by S. Karger AG, Basel } \\
\text { www.karger.com/crg }\end{array}$ \\
\cline { 2 - 3 }
\end{tabular}
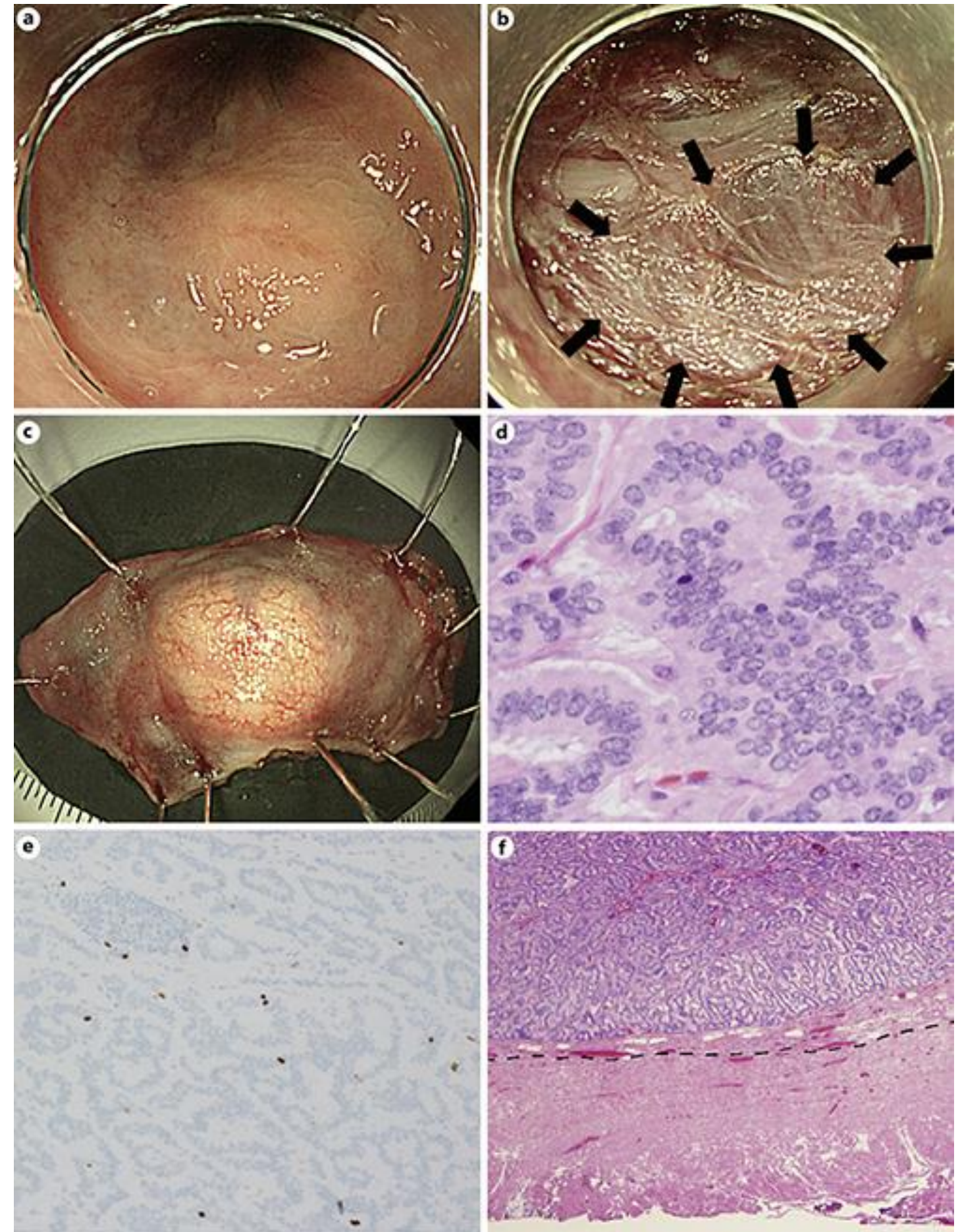
Honjo et al.: Two Cases of Rectal NET Resection Combined with Dissection of the Circular Muscle Layer Using the Endoscopic Submucosal Dissection Technique

Fig. 1. Endoscopic and histopathological findings of Case 1. a An 8-mm SMT was present in the lower rectum on endoscopy. $\mathbf{b}$ Combined resection was applied only to the circular muscle layer in ESD, followed by en bloc resection. The longitudinal muscle layer (arrows) was conserved. c The excised tumor size was 10 $\times 8 \mathrm{~mm}$. d Cells containing a round nucleus and pale cytoplasm in a ribbon-like arrangement were observed on histological examination (high-power field, HE staining). e The Ki-67 index was $\leq 2 \%$, being G1 (high-power field, Ki67 staining). $\mathrm{f}$ The tumor advanced to the submucosal layer, and the circular muscle layer (below the broken line) was concomitantly resected (low-power field, HE staining). SMT, submucosal tumor; ESD, endoscopic submucosal dissection. 


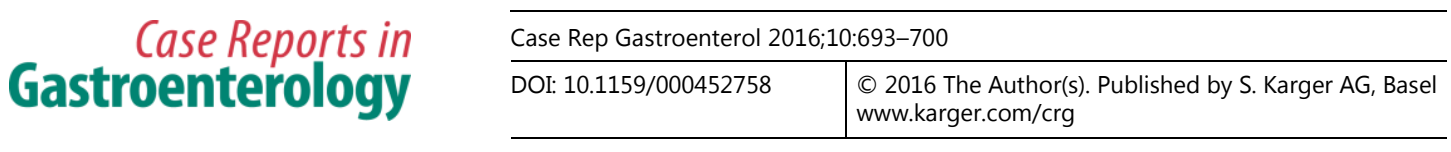 \\ Honjo et al.: Two Cases of Rectal NET Resection Combined with Dissection of the Circular Muscle Layer Using the Endoscopic Submucosal Dissection Technique}
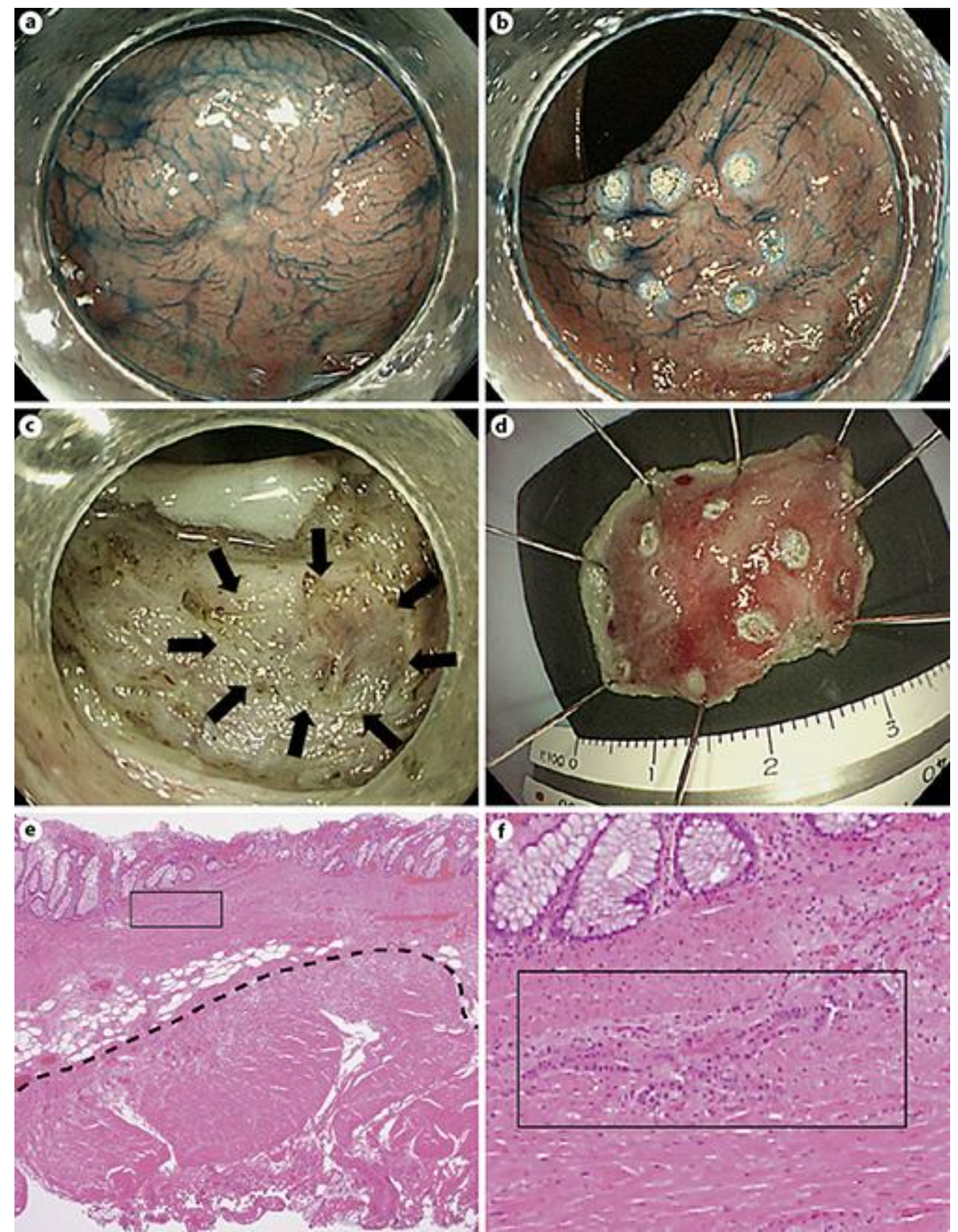

KARGER 
Honjo et al.: Two Cases of Rectal NET Resection Combined with Dissection of the Circular Muscle Layer Using the Endoscopic Submucosal Dissection Technique

Fig. 2. Endoscopic and histopathological findings of Case 2. a An 8-mm scar after EMR was noted in the lower rectum on endoscopy. $\mathbf{b}$ Marking was applied around the lesion. $\mathbf{c}$ Combined resection was applied to the circular muscle layer in ESD, followed by en bloc resection. The longitudinal muscle layer (arrows) was conserved. $\mathbf{d}$ The excised specimen measured $27 \times 15 \mathrm{~mm}$. e Fibrosis of the submucosal layer was noted, and tumorous lesion was present (square). Combined resection of the circular muscle layer (below the broken line) was performed (low-power field, HE staining). $f$ Residual tumorous lesion was present with the diameter of $500 \mu \mathrm{m}$ (square) (high-power field, HE staining). EMR, endoscopic mucosal resection; ESD, endoscopic submucosal dissection. 